Pacific Journal of Mathematics

ON A COINCIDENCE THEOREM OF F. B. FULLER 


\title{
ON A COINCIDENCE THEOREM OF F. B. FULLER
}

\author{
E. FADELL
}

\section{Our objective is the following theorem.}

Theorem (1.1). Let $X$ denote a space dominated by a finite $n$-polyhedron $K$ such that $H^{n}(X ; Z)$ is torsion free. Let $M$ be a compact topological $n$-manifold which is 1-connected and let $f, g: X \rightarrow M$ be two given maps. Then, there is a map $g^{\prime} \sim g: X \rightarrow M$ such that $f$ and $g^{\prime}$ are coincidence free if, and only if, the (rational) Lefschetz coincidence class $L(f, g)=0$.

If $X$ is a finite $n$-polyhedron and $M$ is a triangulated, 1 -connected compact manifold, the theorem is implicit in the work of F. B. Fuller ([5], [6]). In this case, one shows that the obstruction to deforming $f \times g: K \rightarrow M \times M$ to a map $f^{\prime} \times g^{\prime}: K \rightarrow M \times M-\Delta, \Delta$ the diagonal of $M \times M$, is zero. Thus, one obtains maps $f^{\prime} \sim f, g^{\prime} \sim g$, such that $f^{\prime}$ and $g^{\prime}$ are coincidence free. Then, as shown in Fuller's thesis [6], one observes that since $M$ is a manifold (local homogeneity is all that is necessary), deforming both $f$ and $g$ to obtain coincidence free maps is equivalent to deforming just one of them to achieve coincidence freeness. We will use a direct approach, employing general obstruction theory for deforming cross sections into fiber subspaces, simple techniques in fiber spaces to relax the conditions on $X$ and the fact that the classical computations relating $H^{n}(M \times M, M \times M-\Delta)$ and $H^{n}(M \times M)$ where $M$ is a triangulated manifold remain valid for topological manifolds by employing the techniques of tangent fiber spaces [4] or the recent result of Kister [7] that microbundles are bundles. An immediate application of this theorem is an obstruction theory proof of the following converse of the Lefschetz Fixed Point Theorem for compact 1-connected topological manifolds.

COROLlary (1.2). Let $M$ denote a compact 1-connected topological manifold, $f: M \rightarrow M$ a given map and $L_{f}$ the Lefschetz number for $f$. If $L_{f}=0$, there is a map $g, g \sim f$, such that $g$ is fixed point free.

The corollary for triangulated manifolds, because of our previous remarks, is implicit in Fuller [6]. The triangulated case (which is doubtless classical but hard to find explicitly stated anywhere) may also be shown using the theory of Nielson-Reidemeister fixed point classes and results of Wecken [11]. An alternative proof of the corollary in

Received April 20, 1964. This work was supported by the National Science Foundation under Grant NSF-23849 and the Wisconsin Alumni Research Foundation. 
the topological case, without obstruction theory, may be given using Nielson-Reidemeister fixed point classes, results of Weier [12] and the existence of a local index theory for fixed points in the category of ANR's ([2] or [3]). Finally, we remark that (1.1) was discovered by the author before he became aware of Fuller [6] in order to answer a question, raised to him by E. E. Floyd, which is answered by (1.2). (1.2) is easily seen to be false for polyhedra in general.

Singular homology is employed throughout.

2. Preliminaries, We recall first the necessary obstruction theory $[6]$.

Let $\xi=(E, p, B, F), \xi_{0}=\left(E_{0}, p_{0}, B, F_{0}\right)$ denote locally trivial fiber spaces over $B$ such that $E_{0} \subset E, p_{0}=p \mid E_{0}$ and for each $x_{0} \in B$ there is a neighborhood $U$ of $x_{0}$ and a homeomorphism of pairs

$$
\left(p^{-1}(U), p_{0}^{-1}(U)\right) \stackrel{\varphi_{U}}{\longleftarrow}\left(U \times F, U \times F_{0}\right)
$$

such that $p \varphi_{U}(x, y)=x,(x, y) \in U \times F$.

Definition $(2.1) . \quad\left(\xi, \xi_{0}\right)$ is called a locally trivial pair, with fiber $\left(F, F_{0}\right)$.

Suppose now that $\left(\xi, \xi_{0}\right)$ is a locally trivial pair, as above, such that the fibre $\left(F, F_{0}\right)$ is $n$-simple. Then, $\pi_{n}\left(F, F_{0}\right)$ is a local system of groups on $B$. Suppose further that $B$ is a locally finite simplicial complex, with $k$-skeleton denoted by $B^{k}$, and $f: B \rightarrow E$ is a given cross section such that $f\left(B^{n-1}\right) \subset E_{0}$. We paraphrase Theorem 3.2 of [6] as follows.

THEOREM (2.2). There exists an obstruction class

$$
\bar{d}^{n}(f) \in H^{n}\left(B ; \pi_{n}\left(F, F_{0}\right)\right)
$$

such that if $\bar{d}^{n}(f)=0$, then $f$ is homotopic $\left(\right.$ rel $\left.B^{n-2}\right)$ to a cross section $f^{\prime}: B \rightarrow E$ such that $f^{\prime}\left(B^{n}\right) \subset E_{0}$.

REMARK (2.3). It is understood that when cross sections are homotopic we mean homotopic in the family of cross sections.

Suppose now we add the assumption that $\pi_{k}\left(F, F_{0}\right)=0$ for $k<n$ and $\pi_{1}(B)$ acts trivially on $\pi_{n}\left(F, F_{0}\right)$. If $g: B \rightarrow E$ is a given cross section, then by (2.2) there is a cross section $f: B \rightarrow E$, homotopic to $g$, such that $f\left(B^{n-1}\right) \subset E_{0}$. Lundell $\left[6\right.$, p. 167] has computed $\bar{d}^{n}(f)$ as follows. Designate $\pi_{n}\left(F, F_{0}\right)$ by $\pi$. The Hurewicz homomorphism $h: \pi \rightarrow H_{n}\left(F, F_{0}\right)$ is an isomorphism. Let $\theta^{n} \in H^{n}\left(F, F_{0} ; \pi\right)$ denote the fundamental class of $\left(F, F_{0}\right)$, i.e., the class corresponding to $h^{-1}$ under the universal coefficient isomorphism $H^{n}\left(F, F_{0} ; \pi\right) \approx \operatorname{Hom}\left(H_{n}\left(F, F_{0}\right) ; \pi\right)$. Consider the diagram 


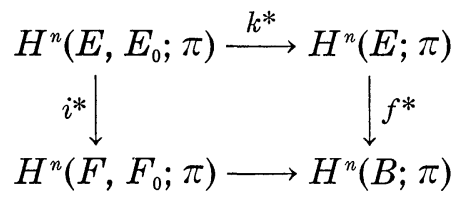

where $i$ and $k$ are inclusion maps.

Proposition (2.4) ([6]). $\bar{d}^{n}(f)=f^{*} k^{*} i^{*-1}\left(\theta^{n}\right)$.

Since $f^{*}=g^{*}, \bar{d}^{n}(f)$ depends only on $g$ and we may employ the notation $\bar{d}^{n}(f)=\bar{d}^{n}(g)$ and state the following.

Proposition (2.5). Given a locally trivial pair $\left(\xi, \xi_{0}\right)$ satisfying all the assumptions imposed above, then a given cross section $g: B \rightarrow E$ is homotopic to a cross section $g^{\prime}: B \rightarrow E$ such that $g^{\prime}\left(B^{n}\right) \subset E_{0}$ if, and only if, $\bar{d}^{n}(g)=g^{*} k^{*} i^{*-1}\left(\theta^{n}\right)=0$.

Let $\xi=(E, p, B, F)$ and $\xi_{0}=\left(E_{0}, p_{0}, B, F_{0}\right)$, where $\left(\xi, \xi_{0}\right)$ is a locally trivial pair. Let $\varphi: K \rightarrow B$ denote a map. Then, $\varphi$ induces a locally trivial pair $\left(\xi^{\prime}, \xi_{0}^{\prime}\right)=\varphi^{-1}\left(\xi, \xi_{0}\right)$ over $K$ in the usual manner as follows. $\xi^{\prime}=\left(E^{\prime}, p^{\prime}, K, F\right)$, where

$$
\begin{gathered}
E^{\prime}=\{(x, y) \in K \times E: \varphi(x)=p(y)\}, \\
p^{\prime}(x, y)=x .
\end{gathered}
$$

$\xi_{0}^{\prime}=\left(E_{0}^{\prime}, p_{0}^{\prime}, K, F_{0}\right)$, where

$$
\begin{gathered}
E_{0}^{\prime}=\left\{(x, y) \in K \times E_{0}: \varphi(x)=p_{0}(y)\right\} \\
p_{0}^{\prime}(x, y)=x .
\end{gathered}
$$

Suppose $g: B \rightarrow E$ is a given cross section in $\xi$. Then, $g^{\prime}(x)=(x, g \varphi(x))$, $x \in K$, defines a corresponding cross section in $g^{\prime}$. Letting $\bar{\varphi}(x, y)=y$ we have the following commutative diagram

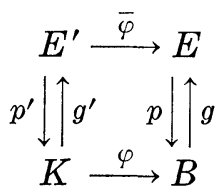

and $\bar{\varphi}\left(E_{0}^{\prime}\right) \subset E_{0}$.

LEMMA (2.6). Suppose $\gamma_{0}: B \rightarrow E_{0}$ is a map (not necessarily a cross section) such that $g \sim \gamma_{0}$ (as maps). If $\left(\xi, \xi_{0}\right)$ is a fibered pair in the sense of [1] (e.g. if $B$ is paracompact), $g$ is homotopic to a cross section $g_{0}: B \rightarrow E_{0}$.

Proof. This lemma is a special case of Lemma (5.1) given in $\S 5$. 
Lemma (2.7). Suppose $\varphi: K \rightarrow B$ is a dominating map, i.e., there is a map $\psi: B \rightarrow K$ such that $\varphi \psi \sim 1$, and $\left(\xi, \xi_{0}\right)$ is a fibered pair. Then, there is a cross section $g_{0}: B \rightarrow E_{0}, g_{0} \sim g$, if, and only if, there is a cross section $g_{0}^{\prime}: K \rightarrow E_{0}^{\prime}, g_{0}^{\prime} \sim g^{\prime}$.

Proof. The necessity is simple and hence we confine ourselves to the sufficiency argument. Suppose, therefore, that there is a cross section $g_{0}^{\prime}: K \rightarrow E_{0}$ such that $g_{0}^{\prime} \sim g^{\prime}$. Let $\gamma=\bar{\phi} g^{\prime} \psi$ and $\gamma_{0}=\bar{\varphi} g_{0}^{\prime} \psi$. Obviously $\gamma \sim \gamma_{0}$. On the other hand, $\gamma=g \varphi \psi \sim g$. Therefore, $g \sim \gamma_{0}$, $\gamma_{0}: B \rightarrow E_{0}$. Lemma (2.6) applies to give the cross section $g_{0}: B \rightarrow E_{0}$, $g_{0} \sim g$.

Continuing with the above notation where $\left(\xi, \xi_{0}\right)$ is locally trivial ficered pair and $g: B \rightarrow E$ is a given cross section let us assume further

(i) $\pi_{i}\left(F, F_{0}\right)=0 i<n, \pi_{n}\left(F, F_{0}\right)$ is simple,

(ii) $\pi_{i}(B)$ acts trivially on $\pi_{n}\left(F, F_{0}\right)$,

(iii) there is a dominating map $\varphi: K \rightarrow B$ where $K$ is a finite polyhedron of dimension $\leqq n$.

We have the following commutative diagram where $\left(\xi^{\prime}, \xi_{0}^{\prime}\right)$ is the locally trivial fibered pair induced over $K$ by $\varphi, g^{\prime}$ is the cross section in $\xi^{\prime}$ associated with $g$ as above, and $\pi=\pi_{n}\left(F, F_{0}\right)$.

(B)

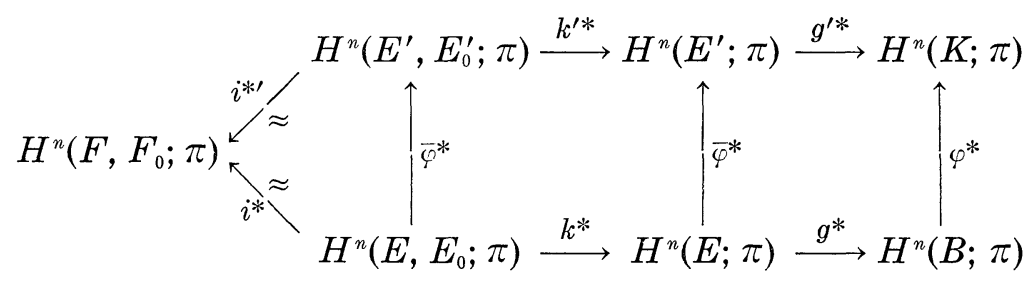

$i, i^{\prime}, k, k^{\prime}$ are inclusion maps.

Proposition (2.8). The cross section $g: B \rightarrow E$ is homotopic to a cross section $g_{0}: B \rightarrow E_{0}$ if, and only if, $g^{*} k^{*} i^{*-1}\left(\theta^{n}\right)=0$, where $\theta^{n}$ is the fundamental class of $\left(F, F_{0}\right)$.

Proof. The assumptions (A) tell us that, via (2.5), the associated cross section $g^{\prime}: K \rightarrow E^{\prime}$ is homotopic to a cross section $g_{0}^{\prime}: K \rightarrow E_{0}^{\prime}$ if, and only if, $\bar{d}^{n}\left(g^{\prime}\right)=g^{\prime *} k^{\prime *} i^{\prime *-1}\left(\theta^{n}\right)=0$. Thus (2.7) implies that there is a cross section $g_{0}: B \rightarrow E_{0}, g \sim g_{0}$ if, and only if, $g^{*} k^{\prime *} i^{\prime *-1}\left(\theta^{n}\right)=0$. But diagram (B) gives

$$
\varphi^{*} g^{*} k^{*} i^{*-1}\left(\theta^{n}\right)=g^{*} k^{*} i^{*-1}\left(\theta^{n}\right) .
$$

Since $\varphi^{*}$ is injective the proposition follows.

The preceding material will be applied to the following situation. 
Let $M$ denote a topological $n$-manifold (connected, separable metric). Let $\xi=(M \times M, p, M, M)$, where $p(b, y)=b$ and

$$
\xi_{0}=\left(M \times M-\Delta, p_{0}, M, M-b\right)
$$

where $\Delta$ is the diagonal in $M \times M$ and $p_{0}$ is the restriction of $p$. Then, $\left(\xi, \xi_{0}\right)$ is a locally trivial fibered pair with fiber $(M, M-b), b \in M$. Let $R$ denote a principal ideal domain and suppose $M$ is $R$-orientable, i.e., $\pi_{1}(M)$ acts trivially on $H_{n}(M, M-b ; R)$ or, alternatively, $\left(\xi, \xi_{0}\right)$ is $R$ orientable. Then, the inclusion map $i:(M, M-b) \rightarrow(M \times M, M \times M-\Delta)$ induces an isomorphism (see [4])

$$
i^{*}: H^{n}(M \times M, M \times M-\Delta ; R) \stackrel{\approx}{\longrightarrow} H^{n}(M, M-b ; R) \approx R .
$$

Suppose now that $f: X \rightarrow M$ is any given map where $X$ is an arbitrary space. Let $E=X \times M, E_{0}=X \times M-\Delta_{f}$, where $\Delta_{f}$ is the graph of $f$. Let $q: E \rightarrow X$ be projection on the first factor and $q_{0}=$ $q \mid E_{0}$. For fixed $x \in X, q^{-1}(x)=x \times M=M$ and $q_{0}^{-1}(x)=x \times(M-f(x))=$ $M-f(x)$. Let $\xi(f)=(E, q, X, M), \xi_{0}(f)=\left(E_{0}, q_{0}, X, M-f(x)\right)$.

Proposition (2.9). $\left(\xi(f), \xi_{0}(f)\right)$ is a locally trivial fibered pair with fiber $(M, M-f(x))$. If $\left(\xi, \xi_{0}\right)$ is $R$-orientable, so is $\left(\xi(f), \xi_{0}(f)\right)$.

Proof. $\left(\xi(f), \xi_{0}(f)\right)$ may be identified with $f^{-1}\left(\xi, \xi_{0}\right)$.

REMARK (2.10)。 There is a natural map $\bar{f}: E=X \times M \rightarrow M \times M$ given by $\bar{f}(x, y)=(f(x), y)$ which gives rise to a commutative diagram

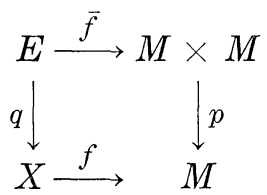

and $\bar{f}\left(E_{0}\right)=\bar{f}\left(X \times M-\Delta_{f}\right) \subset M \times M-\Delta$.

If $M$ is 1 -connected and $\operatorname{dim} M \geqq 3$, then $M-b$ is 1 -connected and $\pi_{k}(M, M-b)=0$ for $k<n$ and $\pi_{n}(M, M-b)$ is a simple system. If furthermore, $X$ is dominated by a finite polyhedron of dimension $\leqq n$, then $\left(\xi(f), \xi_{0}(f)\right)$ satisfies the conditions in (A).

REMARK (2.11). If $M$ is a compact, 1-connected 2-manifold, then $M=S^{2}$ (the 2-sphere) and the preceding remarks remain valid.

3. Some computations. We discuss first Lefschetz coincidence theory in this setting.

Let $M$ denote an orientable ( $=Z$-orientable) compact topological 
$n$-manifold, $R$ any principal ideal domain. Let $\mu \in H^{n}(M ; R), \bar{\mu} \in H_{n}(M ; R)$ denote fundamental classes corresponding to a given orientation of $M$. Then for $b \in M$, the inclusion map $j: M \rightarrow(M, M-b)$ induces an isomorphism [8]

$$
H^{n}(M ; R) \stackrel{j^{*}}{\approx} H^{n}(M, M-b ; R)
$$

and we define $U_{b}$ by $j^{*}\left(U_{b}\right)=\mu$. Now consider the fibered pair $\left(\xi, \xi_{0}\right)$ where $\xi=(M \times M, p, M, M), \xi_{0}=\left(M \times M-\Delta, p_{0}, M, M-b\right)$ (see $\left.\S 2\right)$ which gives rise to the diagram

$$
H^{n}(M, M-b ; R) \stackrel{i^{*}}{\approx} H^{n}(M \times M, M \times M-\Delta ; R) \stackrel{k^{*}}{\longrightarrow} H^{n}(M \times M ; R),
$$

where $i$ and $k$ are inclusions. Define $U$ by $i^{*}(U)=U_{b}$ and set $\underline{U}=$ $k^{*}(U)$. This class $\underline{U}$ is studied by Milnor [7] in case $R$ is a field and $M$ is an $R$-orientable differentiable manifold. The techniques carry over to the topological situation by using the techiques of tangent fiber spaces [4] or a recent result of J. Kister [7] and we will make use of this fact. For convenience we refer to $\underline{U}$ as the basic $R$-class for $M$. It is well-defined up to orientation

Definition (3.1). Let $M$ denote an $R$-orientable compact topological manifold with basic $R$-class $\underline{U}$. Let $f, g: X \rightarrow M$ denote two maps from a space $X$ to $M$. These maps induce $f \times g: X \times X \rightarrow M \times M$. Furthermore, let $\delta: X \rightarrow X \times X$ denote the diagonal map. The class

$$
\delta^{*}(f \times g)^{*}(\underline{U})=L(f, g ; R)
$$

is called the $R$-Lefschetz coincidence class for $f$ and $g$.

REMARK (3.2). When $R=Q$, the field of rationals, $L(f, g ; Q)$ is the Lefschetz class usually considered and we distinguish it by the notation $L(f, g)$.

The following theorem in which we employ the notation in (3.1) is immediate.

THEOREM (Lefschetz). If $f$ and $g$ are coincidence free, then $L(f, g ; R)=0$.

Let us assume now that $R$ is a field and $M$ is $R$-orientable as above. Following Milnor [7] arrange a basis

$$
1=\alpha_{1}, \alpha_{2}, \cdots, \alpha_{N}=\mu .
$$

for $H^{*}(M ; R)$ in increasing order of dimension. We find it also convenient to introduce the notation 
(2) $K_{p}=$ integer subscripts in (1) of $p$-dimensional elements.

Then, the basic $R$-class $\underline{U}$ takes on the form

$$
\underline{U}=\Sigma c_{i j} \alpha_{i} \times \alpha_{j}=\sum_{p}\left(\sum_{i \in K_{p}} \sum_{j \in K_{n-p}} c_{i j} \alpha_{i}^{p} \times \alpha_{j}^{n-p}\right),
$$

where the matrix

$$
C_{p, n-p}=\left\|c_{i j}\right\|, \quad(i, j) \in K_{p} \times K_{x-p}
$$

is square and if

$$
Y_{n-p, p}=\left\|y_{i j}\right\|, \quad y_{i j}=\left\langle\alpha_{i} \cup \alpha_{j}, \bar{\mu}\right\rangle, \quad(i, j) \in K_{n-p} \times K_{p},
$$

we have

$$
(-1)^{n p} C_{p, n-p} Y_{n-p, p}=I \text { (an identity matrix) . }
$$

Define

$$
\beta_{j}^{n-p}=(-1)^{p} \sum_{k \in K_{n-p}} c_{j_{k}} \alpha_{k}^{n-p}, \quad j \in K_{p} .
$$

Then it is easily verified that

$$
\left\langle\alpha_{i}^{p} \cup \beta_{j}^{n-p}, \bar{\mu}\right\rangle=\delta_{i j}, \quad i, j \in K_{p},
$$

so that the $\beta$ 's form a dual basis. A simple computation now gives the classical formula

$$
L(f, g ; R)=\sum_{p}\left((-1)^{p} \sum_{i \in K_{p}} f^{*}\left(\alpha_{i}^{p}\right) \cup g^{*}\left(\beta_{\imath}^{n-p}\right)\right)
$$

where $f, g$ are as in (3.1). If $X=M, f=1$, and $R$ is the field of rationals, another straightforward computation shows

$$
L(1, g)=(-1)^{n}\left(\sum_{p}(-1)^{p} \operatorname{tr}\left(g_{p}^{*}\right)\right) \mu=(-1)^{n} L_{g} \mu
$$

where $\operatorname{trg} g_{p}^{*}$ is the trace of $g^{*}$ in dimension $p$ and $L_{q}=\sum_{p}(-1)^{p} \operatorname{tr}\left(g_{p}^{*}\right)$ is the usual Lefschetz number for $g$.

REMARK (3.2). It is clear that the preceding material is applicable to compact topological manifolds which are not necessarily orientable if $Z_{2}$ coefficients are employed.

4. Proof of Theorem (1.1). The necessity is immediate and we confine ourselves to the "if part."

Let $\left(\xi, \xi_{0}\right)$ denote the fibered pair in $\S 3$, i.e., $\xi=(M \times M, p, M, M)$, $\xi_{0}=\left(M \times M-\Delta, p_{0}, M, M-b\right)$. The given map $f: X \rightarrow M$ induces the fiber space $\left(\xi(f), \xi_{0}(f)\right)$ over $X(\S 2)$ which satisfies conditions (A) 
(§2). The fiber of $\left(\xi(f), \xi_{0}(f)\right)$ over $x \in X$ is $(M, M-b), b=f(x)$. Letting $\xi(f)=(E, q, X, M), \xi_{0}(f)=\left(E_{0}, q_{0}, X, M-b\right)$, we have the commutative diagram

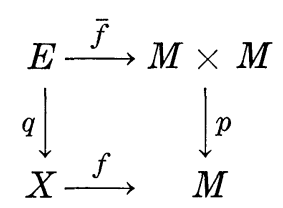

where $\bar{f}(x, y)=(f(x), y)$. The other given map $g: X \rightarrow M$ induces a cross section $\widetilde{g}: X \rightarrow E$ by $\widetilde{g}(x)=(x, g(x)), x \in X$. We note at this point that $\bar{f} \widetilde{g}=(f \times g) \delta$, where $\delta: X \rightarrow X \times X$ is the diagonal map and $f \times g: X \times X \rightarrow M \times M$ is the product map. Our objective is to deform the cross section $\widetilde{g}$ into $E_{0}=X \times M-\Delta_{f}$. Consider the commutative diagram

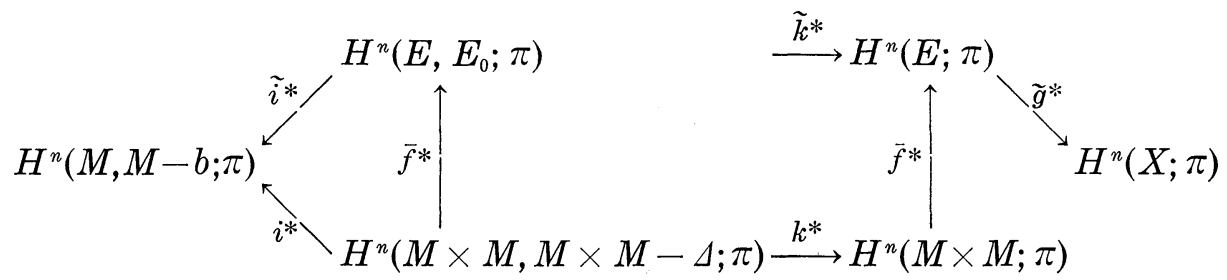

where $i, \tilde{i}, k, \tilde{k}$ are inclusions and $\pi=\pi_{n}(M, M-b) \approx Z$. If $\theta^{n}$ is the fundamental class of $(M, M-b)$, we need to show, by (2.8), that $\widetilde{g}^{*} \widetilde{k}^{*} \widetilde{i}^{*-1}\left(\theta^{n}\right)=0$. We may choose an orientation $\mu \in H^{n}(M ; \pi)$ of $M$ so that $j^{*}\left(\theta^{n}\right)=\mu$ where $j: M \rightarrow(M, M-b)$ is the inclusion map. This orientation determines

$$
U \in H^{n}(M \times M, M \times M-\Delta ; \pi) \quad \text { and } \quad \underline{U} \in H^{n}(M \times M ; \pi)
$$

such that $\underline{U}=k^{*} i^{*-1}\left(\theta^{n}\right)$. The corresponding $\pi$-Lefschetz class is given by $\delta^{*}(f \times g)^{*}(\underline{U})=L(f, g ; \pi)$. On the other hand

$$
\begin{aligned}
\widetilde{g}^{*} \widetilde{k}^{*} \widetilde{i}^{*-1}\left(\theta^{n}\right) & =\widetilde{g}^{*} \widetilde{k}^{*} \widetilde{i}^{*-1} i^{*}(U)=\widetilde{g}^{*} \widetilde{k}^{*} \bar{f}^{*}(U) \\
& =\widetilde{g}^{*} \bar{f}^{*} k^{*}(U)=\widetilde{g} \bar{f}^{*}(\underline{U})=\delta^{*}(f \times g)^{*}(\underline{U}) .
\end{aligned}
$$

Therefore, $L(f, g ; \pi)=\widetilde{g}^{*} \widetilde{k}^{*} i^{*-1}\left(\theta^{n}\right)$ and hence the integral Lefschetz class is precisely the obstruction class in question. Now, if $Q$ is the field of rationals, the inclusion homomorphism $l: \pi \rightarrow Q$ induces $l^{*}: H^{n}(X ; \pi) \rightarrow H^{n}(X ; Q)$. It is clear that $l^{*}(L(f, g ; \pi))=L(f, g ; Q)=$ $L(f, g)$, where the Lefschetz class $L(f, g)$ is chosen in terms of the orientation $\mu$. But the assumption that $H^{n}(X ; \pi)$ is torsion free together with the fact that $X$ is dominated by a finite polyhedron implies that $l^{*}$ is injective. Therefore, $L(f, g)=0$ implies $L(f, g ; \pi)=0$, which in turn implies $\widetilde{g} \widetilde{k}^{*} \tilde{i}^{*-1}\left(\theta^{n}\right)=0$ and the theorem follows. 
Since a compact topological $n$-manifold is always dominated by a finite polyhedron of dimension $n$, we have the following corollary, which is a converse to the Lefschetz Fixed Point Theorem.

CoRollary (4.1). Let $M$ denote a compact, 1-connected topological manifold, $f: M \rightarrow M$ a given map and $L_{f}$ the Lefschetz number for $f$. If $L_{f}=0$, there is a map $g, g \sim f$, such that $g$ is fixed point free.

Proof. By (10) of $\S 3, L(1, f)=(-1)^{n} L_{f} \mu$, where $n=\operatorname{dim} M$. If $L_{f}=0$, then $L(1, f)=0$ and $f \sim g$ such that 1 and $g$ have no coincidences, i.e., $g$ has no fixed points.

Corollary (4.1) may be reformulated as follows. Let $\alpha$ denote a homotopy class of maps $M \rightarrow M$. Call $\alpha$ fixed point free if $\alpha$ contains a fixed point free representative Also define $L_{\alpha}$ by $L_{\alpha}=L_{f}, f \in \alpha$.

COROLlary (4.2). If $M$ is a compact, 1-connected topological manifold and $\alpha: M \rightarrow M$ is a homotopy class of maps, then $\alpha$ is fixed point free if, and only if, $L_{\alpha}=0$.

\section{Appendix.}

LEMma (5.1). Let $\left(\xi, \xi_{0}\right)$ denote a locally trivial fibered pair where $\xi=(E, p, B, F), \xi_{0}=\left(E_{0}, p_{0}, B, F_{0}\right)$. Suppose we are given a commutative diagram of maps

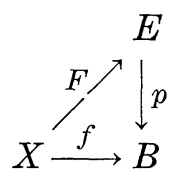

and a map $G: X \rightarrow E_{0}$ such that $F \sim G$. Then, there is a homotopy $H: X \times I \rightarrow E$ such that $H_{1}=F, p H_{t}=f, 0 \leqq t \leqq 1$, and $H_{0}: X \rightarrow E_{0}$.

Proof. Since $\left(\xi, \xi_{0}\right)$ is a fibered pair in the sense of [1], there is a regular lifting function $\lambda$ for $\xi$ which works simultaneously for $\xi_{0}$, i.e., if $e_{0} \in E_{0}$ and $\omega \in B^{I}$, then $\lambda\left(e_{0}, \omega\right)$ is a path in $E_{0}$. By assumption there is a homotopy $K: X \times I \rightarrow E$ such that $K_{0}=G$ and $K_{1}=F . \quad K$ induces a map $\widetilde{K}: X \rightarrow E^{I}$ in the usual manner with $\widetilde{K}(x)(t)=K(x, t)$, $0 \leqq t \leqq 1$. Let $\alpha(x) \in B^{I}$ denote the path $p(x)$, and observe that $\alpha(x)(t)=p(K(x, t)), 0 \leqq t \leqq 1$, and, in particular, $\alpha(x)(1)=f(x)$. For $0 \leqq s \leqq t$, let $\alpha(x)_{s}$ denote the path given by $\alpha(x)_{s}(t)=\alpha(x)(s+t-s t)$, $0 \leqq t \leqq 1 . \alpha(x)_{s}$ is a path with initial point $p(H(x, s))$ and terminal point $f(x)$. The required homotopy $H$ is given by

$$
H(x, s)=\lambda\left[K(x, s) \alpha(x)_{s}\right](1), \quad 0 \leqq s \leqq 1 .
$$




\section{Bibliography}

1. G. Allaud and E. Fadell, $A$ fiber homotopy extension theorem, Trans. Amer. Math. Soc. 104 (1962), 239-251.

2. D. G. Bourgin, Modern Algebraic Topology, MacMillan (1963).

3. F. E. Browder, On the fixed point index for continuous mappings of locally connected spaces, Summa Brasil. Math. 4 (1960), 253-293.

4. E. Fadell, Generalized normal bundless for locally flat imbeddings, to appear in Trans. Amer. Math. Soc. 114 (1965), 488-513.

5. F. B. Fuller, The homotopy theory of coincidences, Ann. of Math. 59 (1954), 219226.

6. - The Homotopy Theory of Coincidences, Princeton Thesis, 1951.

7. J. Kister, Microbundles are bundles, Ann. Math. 80(1964), 190-199.

8. A. T. Lundell, Obstruction theory of principal fiber bundles, Trans. Amer. Math. Soc. 97 (1960), 161-192.

9. J. Milnor, Lectures on Characteristic Classes (Mimeographed Notes), Princeton, 1957.

10. D. Puppe, Topologie II, Universität Bonn, 1960.

11. F. Wecken, Fixpunktklassen III, Math. Annalen 118 (1941-43), 544-577.

12. J. Weier, Fixpunkttheorie in topologischen Mannigfaltigkeiten, Math. Zeit. 59 (1953), 171-190.

The Institute for Advanced Study 


\section{PACIFIC JOURNAL OF MATHEMATICS}

\section{EDITORS}

\author{
H. Samelson \\ Stanford University \\ Stanford, California \\ R. M. Blumenthal \\ University of Washington \\ Seattle, Washington 98105
}

\author{
J. Dugundu \\ University of Southern California \\ Los Angeles, California 90007 \\ *Richard Arens \\ University of California \\ Los Angeles, California 90024
}

\section{ASSOCIATE EDITORS}
E. F. BeCKENBACH
B. H. NeUMANN
F. WOLF
K. YosidA

\section{SUPPORTING INSTITUTIONS}

\author{
UNIVERSITY OF BRITISH COLUMBIA \\ CALIFORNIA INSTITUTE OF TECHNOLOGY \\ UNIVERSITY OF CALIFORNIA \\ MONTANA STATE UNIVERSITY \\ UNIVERSITY OF NEVADA \\ NEW MEXICO STATE UNIVERSITY \\ OREGON STATE UNIVERSITY \\ UNIVERSITY OF OREGON \\ OSAKA UNIVERSITY \\ UNIVERSITY OF SOUTHERN CALIFORNIA
}

\author{
STANFORD UNIVERSITY \\ UNIVERSITY OF TOKYO \\ UNIVERSITY OF UTAH \\ WASHINGTON STATE UNIVERSITY \\ UNIVERSITY OF WASHINGTON \\ * * * * \\ AMERICAN MATHEMATICAL SOCIETY \\ CALIFORNIA RESEARCH CORPORATION \\ SPACE TECHNOLOGY LABORATORIES \\ NAVAL ORDNANCE TEST STATION
}

Mathematical papers intended for publication in the Pacific Journal of Mathematics should by typewritten (double spaced). The first paragraph or two must be capable of being used separately as a synopsis of the entire paper. It should not contain references to the bibliography. No separate author's resumé is required. Manuscripts may be sent to any one of the four editors. All other communications to the editors should be addressed to the managing editor, Richard Arens, at the University of California, Los Angeles, California 90024.

50 reprints per author of each article are furnished free of charge; additional copies may be obtained at cost in multiples of 50 .

The Pacific Journal of Mathematics is published quarterly, in March, June, September, and December. Effective with Volume 13 the price per volume (4 numbers) is $\$ 18.00$; single issues, $\$ 5.00$. Special price for current issues to individual faculty members of supporting institutions and to individual members of the American Mathematical Society: $\$ 8.00$ per volume; single issues $\$ 2.50$. Back numbers are available.

Subscriptions, orders for back numbers, and changes of address should be sent to Pacific Journal of Mathematics, 103 Highland Boulevard, Berkeley 8, California.

Printed at Kokusai Bunken Insatsusha (International Academic Printing Co., Ltd.), No. 6, 2-chome, Fujimi-cho, Chiyoda-ku, Tokyo, Japan.

PUBLISHED BY PACIFIC JOURNAL OF MATHEMATICS, A NON-PROFIT CORPORATION

The Supporting Institutions listed above contribute to the cost of publication of this Journal, but they are not owners or publishers and have no responsibility for its content or policies.

* Basil Gordon, Acting Managing Editor until February 1, 1966. 


\section{Pacific Journal of Mathematics}

\section{Vol. 15, No. $3 \quad$ November, 1965}

David R. Arterburn and Robert James Whitley, Projections in the space of

bounded linear operators .................................

Robert McCallum Blumenthal, Joram Lindenstrauss and Robert Ralph Phelps,

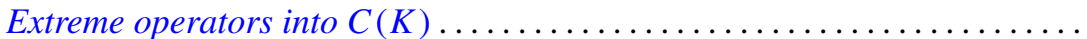

L. Carlitz, A note on multiple exponential sums ................... 757

Joseph A. Cima, A nonnormal Blaschke-quotient .................... 767

Paul Civin and Bertram Yood, Lie and Jordan structures in Banach algebras . . . 775

Luther Elic Claborn, Dedekind domains: Overrings and semi-prime

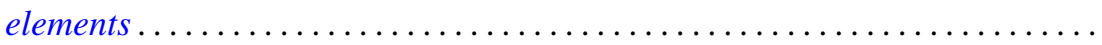

799

Luther Elic Claborn, Note generalizing a result of Samuel's .............. 805

George Bernard Dantzig, E. Eisenberg and Richard Warren Cottle, Symmetric

dual nonlinear programs ................................... 809

Philip J. Davis, Simple quadratures in the complex plane ............... 813

Edward Richard Fadell, On a coincidence theorem of F. B. Fuller ............ 825

Delbert Ray Fulkerson and Oliver Gross, Incidence matrices and interval

graphs ........................................ 835

Larry Charles Grove, Tensor products over $H^{*}$-algebras ..................

Deborah Tepper Haimo, $L^{2}$ expansions in terms of generalized heat polynomials

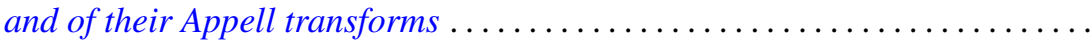

I. Martin (Irving) Isaacs and Donald Steven Passman, A chardcterization of groups in terms of the degrees of their characters ..........

Donald Gordon James, Integral invariants for vectors over local fields........ 905

Fred Krakowski, A remark on the lemma of Gauss ................... 917

Marvin David Marcus and H. Minc, A subdeterminant inequality ........... 921

Kevin Mor McCrimmon, Norms and noncommutative Jordan algebras ........ 925

Donald Earl Myers, Topologies for Laplace transform spaces ............... 957

Olav Njstad, On some classes of nearly open sets .................... 961

Milton Philip Olson, A characterization of conditional probability ........... 971

Barbara Osofsky, A counter-example to a lemma of Skornjakov .............. 985

Sidney Charles Port, Ratio limit theorems for Markov chains ............... 989

George A. Reid, A generalisation of $W^{*}$-algebras ...................... 1019

Robert Wells Ritchie, Classes of recursive functions based on Ackermann's

function ........................................... 1027

Thomas Lawrence Sherman, Properties of solutions of $n$th order linear

differential equations ........................................ 1045

Ernst Snapper, Inflation and deflation for all dimensions . .............. 1061

Kondagunta Sundaresan, On the strict and uniform convexity of certain Banach

spaces............................................. 1083

Frank J. Wagner, Maximal convex filters in a locally convex space .......... 1087

Joseph Albert Wolf, Translation-invariant function algebras on compact 